\title{
La diplomacia panda y el caso de México
}

\section{Panda Diplomacy and the case of Mexico}

DOI: $10.32870 /$ mycp.v8i23.585

\author{
Carlos Cerda Dueñas ${ }^{1}$
}

\section{Resumen}

En este artículo se analiza la figura de la diplomacia pública denominada "diplomacia panda" que es exclusiva de China y cómo ha servido a este país este vehículo del llamado poder blando. Empleando el método de análisis de datos, se examinan cuestiones teóricas respecto de la diplomacia pública; se revisa la diplomacia panda como vertiente de la diplomacia pública china y su utilización en la época contemporánea y, finalmente, se analiza el ejercicio de la diplomacia panda en el caso de México, concluyendo que se aplicó bajo el supuesto de que este país serviría de punta de lanza para el reconocimiento de otros países de la región, lo que necesariamente no ocurrió, y que la actual administración china ha retomado esta herramienta para lograr el acercamiento y eventuales concesiones de parte de los países interesados en obtener y ostentar pandas.

Palabras clave: diplomacia pública, diplomacia panda, poder blando, China, México.

\begin{abstract}
This article analyzes the tool of public diplomacy called "panda diplomacy", exclusive of China and how this piece of what is called soft power has served to this country. Using the method of data analysis, examines theoretical issues regarding public diplomacy; reviews panda diplomacy as an aspect of Chinese public diplomacy and its current use and, finally, analyzes the case of diplomacy panda with respect to Mexico. It concludes that this country would be of spearhead for recognition of other countries in the region, which necessarily did not take place. Another conclusion is that Chinese administration has resumed this tool to achieve rapprochement and to get possible concessions from countries interested in having pandas.
\end{abstract}

Keywords: public diplomacy, panda diplomacy, soft power, China, Mexico.

\section{Introducción}

De acuerdo con Anderlini (2017), en los últimos años la diplomacia panda ha cambiado claramente del énfasis de la conservación hacia el simbolismo político del panda y que, evidentemente, se ha convertido en un vehículo muy explícito para el poder blando. Poder blando que en ocasiones es llamado

Artículo recibido el 27 de noviembre de 2018 y dictaminado el 18 de enero de 2019.

1. Tecnológico de Monterrey, Campus Guadalajara, Escuela de Ciencias Sociales y Gobierno. . Av. Ramón Corona 2514, C.P. 45138, Zapopan, Jalisco, México. ORCiD: http://orcid.org/00000001-6860-1943 Correo electrónico: carlos.cerda@tec.mx 
poder suave y que corresponde al término inglés de soft power, es decir, una herramienta para conseguir objetivos de otros países sin el uso de la fuerza.

Al ser el panda una especie única de China, le convierte en una modalidad de la diplomacia pública exclusiva que este país ha sabido utilizar a lo largo del tiempo, pero que en el gobierno de Xi Jinping ha cobrado nuevamente un vigor que había perdido, sobre todo por cuestiones de conservación de la especie que, precisamente, se ha convertido ahora en el vehículo que le permite a China obtener ingresos económicos y políticos.

En este artículo se analiza la diplomacia panda como una modalidad de la diplomacia pública y del llamado poder blando, así como su uso por parte del gobierno chino, particularmente en la actual administración. Se analiza también el caso de México, revisando el contexto y las causas que pudieron ser el origen de que este país sea el único latinoamericano seleccionado por los chinos para la práctica de la "diplomacia panda".

\section{La diplomacia pública: reflexiones teóricas}

Joseph Nye señala que poder blando significa "conseguir que otros quieran los resultados que quien ostenta el poder quiere, y el poder blando copta en lugar de coaccionar a otros" (Nye, 2010 p. 117), es decir, obtener lo que se quiere, por lo tanto es la capacidad de influir en el comportamiento de los demás para obtener los resultados que uno desea y para lograrlo existen dos opciones: hacerlo por medio de la coerción en función del poder militar o económico (poder duro), o de la atracción, por medio de la cultura, los valores, ideales y política exterior (poder blando). Respecto de la política exterior, Nye señala que requiere legitimación internacional y una forma de lograrlo es con el cultivo de una imagen positiva de esa nación, y añade que el poder blando de un país depende de muchos factores, incluyendo su desempeño, imagen globaly reputación internacional y que un Estado puede utilizar el poder blando para atraer a simpatizantes y socios hacia sus políticas, puntos de vista y acciones (Nye, 2010 pp. 123-127); esto implica una expresión de la capacidad que tiene un actor de obtener lo que quiere en el entorno internacional a causa del atractivo de su cultura, más que por la influencia militar o económica.

En la promoción de esa imagen positiva cumple un papel fundamental la llamada diplomacia pública. Para académicos y muchos encargados de instrumentar la política exterior, la diplomacia pública forma parte del poder blando, es decir, se trata de una forma indirecta de ejercer el poder mediante el 
convencimiento y la aceptación de valores para impulsar propuestas y legitimar acciones de política exterior (García de Alba, 2009). El prestigio y la buena reputación siguen teniendo una importancia fundamental en la estrategia de política exterior y en la posibilidad de tener éxito en su consecución, por ello la imagen que un país proyecta hacia el exterior y el modo en que el resto le percibe constituye hoy en día una cuestión ineludible y de capital importancia en el ámbito de las relaciones internacionales, tanto políticas como económicas y sociales (Espinosa de los Monteros, 2012).

Es indudable que una nueva visión debe ver la política exterior menos como una interacción entre las élites de los gobiernos y más como una multifacética interacción entre las sociedades, en la que los gobiernos, entre otros actores, desempeñan un importante papel en el impulso del diálogo y en el avance hacia resultados a través de intercambios difusos en un número de niveles. Esto no significa que los gobiernos no tengan políticas relativas, se entrevisten con líderes extranjeros, muevan sus ejércitos ni negocien tratados internacionales. No significa tampoco que el poder militar sea menos crucial. Cada una de estas funciones tradicionales de gobierno tendrá que ser desarrollada tomando en cuenta la opinión pública internacional y más ampliamente el contexto internacional definido y presentado en una forma y manera que utilice cualquier medio para mejor alcanzar a las audiencias (Metzl, 1999). En este sentido, la diplomacia pública es el intento de un actor internacional de gestionar el entorno internacional mediante el compromiso con un público extranjero. En el plano histórico, la diplomacia pública ha adquirido la forma de contacto entre un gobierno y la población de otro Estado (Cull, 2009), es decir, el concepto de diplomacia pública ahora se refiere también a las labores que realizan los gobiernos para generar información destinada a explicar sus políticas a ciudadanos de otras naciones. A diferencia de la diplomacia tradicional, que se orienta concretamente a los gobiernos y organizaciones, o sea, primordialmente a sujetos de derecho internacional convencionales, la diplomacia pública tiene como objetivo la sociedad civil (García de Alba, 2009), pero también es cierto que esa sociedad puede contribuir en la diplomacia pública, por lo tanto, no es exclusiva de los Estados; sin embargo, el enfoque en este artículo está orientado a la que realizan estos. 
La forma de concebir la diplomacia pública no es unánime, ${ }^{2}$ como tampoco son uniformes los mecanismos que emplean los actores que la ejercen y las facetas que se adoptan son cada vez más variadas: desde la tradicional diplomacia cultural, como la deportiva, económica y corporativa o empresarial, pasando ahora a la desarrollada a través de las nuevas tecnologías en lo que se denomina diplomacia digital; o la ya referida diplomacia de los ciudadanos.

Algunos países, al tener un rasgo distintivo único, se valen de éste para desplegar una faceta de la diplomacia pública que les es exclusiva; por ejemplo, el contar con especies animales autóctonas, han utilizado a estos animales para desarrollar diplomacia pública y en ésta se inscribe la llamada diplomacia panda.

El uso de animales emblemáticos en la diplomacia tiene una larga historia, pero expresiones un tanto recientes las podemos observar cuando, en la década de los ochenta, Indonesia dotó de cuatro dragones de Komodo para el ex primer ministro de Singapur, Lee Kuan Yew. El ex presidente George H. W. Bush también recibió un lagarto como un regalo de Indonesia, así como el gobierno de Mongolia ha regalado caballos a los dignatarios visitantes.

El despliegue diplomático de Australia de animales nativos (particularmente el koala y el ornitorrinco) a otros países se convirtió en una cuestión formal durante la Segunda Guerra Mundial. El atractivo popular y el interés científico en el ornitorrinco lo convirtieron en un regalo diplomático altamente valorado. El primer ornitorrinco diplomático de Australia dejó Melbourne en 1943, viajó a Inglaterra navegando en el Phillip M. V. También en Australia, en 1983, el entonces ministro de turismo federal, John Brown, hizo su mejor esfuerzo para desasociar la imagen en el extranjero de Australia sobre el koala, declarando que los turistas iban a descubrir que estaban llenos de pulgas, pero dos generaciones más tarde la opinión de Brown ha sido olvidada. La diplomacia koala nunca ha sido más grande. En noviembre de 2014 durante la Cumbre del G20 en Brisbane, los líderes mundiales participantes posaron uno por uno con un complaciente koala. En 2015, cuatro hembras koala debutaron frente al público en el zoológico de Singapur. Los koalas llegaron en calidad de préstamo de Australia para conmemorar el 50 aniversario de la soberanía de Singapur, así como los 50 años de relaciones diplomáticas con Australia.

2. Por ejemplo, el Ministerio de Relaciones Exteriores de Brasil señala que si bien el concepto de diplomacia pública ha estado asociado a la promoción de la imagen de un país en el exterior, en Brasil no sólo se entiende bajo esa acepción sino también en el sentido de mayor apertura del Ministerio y de la política exterior brasileña a la sociedad civil en un esfuerzo de democratizar y transparentar las políticas nacionales de esta materia (Itamaraty, 2015). 
Los políticos que visitan Australia son regularmente llevados a cargar en sus brazos a un koala (Russo, 2016).

Con los mismos objetivos, pero con una variada gama de circunstancias particulares y épocas distintas, China ha utilizado la diplomacia panda, aprovechando la ventaja de ser el único país en el mundo en contar con esta especia osuna (Ailuropoda melanoleuca) y la poderosa atracción que ejerce en el público, es decir, China busca atraer al público extranjero para mejorar su percepción y ejercer influencia en determinados gobiernos con el préstamo, o en su momento donación de ejemplares de esta especie.

\section{La diplomacia panda como vertiente de la diplomacia pública china}

La diplomacia panda es un instrumento de diplomacia pública que permite que el gobierno chino llegue a audiencias en el extranjero con el objetivo de proyectar una imagen nacional positiva de China e incrementar su publicidad entre el público extranjero. La diplomacia panda, mediante el envío de pandas como gesto de buena voluntad y símbolo de la amistad, sirve para dar a China un alto perfil en países específicos y capturar a las audiencias en el extranjero interesadas en China. Interviniendo de forma positiva en la opinión pública extranjera con metas a corto plazo, la diplomacia panda también contribuye a obtener objetivos diplomáticos de largo y mediano plazos de China en el país de destino (Xing, 2010 p. 19).

Si bien se ha establecido que ya desde la dinastía Tang (618-907 d. C.), las cortes imperiales de China obsequiaron pandas a sus países vecinos, la caracterización y conceptualización de lo que se conoce hoy como diplomacia panda se remonta al periodo de la Segunda Guerra Mundial y se han elaborado diversas clasificaciones de las etapas en que se ha desarrollado (Lin, 2009; Nicholls, 2010; Xing, 2010; Buckingham, Neil, David \& Jepson, 2013; Anderlini, 2017, entre otros), pero la mayor coincidencia entre éstas es determinarla de acuerdo con el esquema en que los pandas han sido entregados: donación, préstamo con fines de lucro o préstamo con fines de investigación o conservación.

En tiempos modernos habría que remontarse al uso que de esta herramienta se hizo por parte de la República de China: la primera vez que China entregó un panda en nombre del gobierno a otro país fue en 1941 cuando Soong May-ling (también conocida como Madame Chiang Kai-shek, por ser la esposa del presidente Chiang Kai-shek) obsequió un par de pandas a los 
estadounidenses, llamados Pan-Dee y Pan-Dah, para el Zoológico del Bronx como una forma de agradecer a Estados Unidos la ayuda durante la guerra en su lucha contra la ocupación japonesa. El gobierno de Chiang dio en total 14 ejemplares a países occidentales.

Con el surgimiento de la República Popular China, Mao Zedong continuó con esta práctica, pero enfocada hacia aliados elegidos de la órbita socialista: en 1957 y 1959 China dio dos pandas, Ping-Ping y An-An, como regalo a Moscú. En ese sentido, los pandas fueron herramientas para confirmar la proximidad ideológica y de fortalecimiento de las relaciones dentro del campo socialista (Szczudlik, 2017). Corea del Norte fue otro de los beneficiarios de este esquema, pues recibió un total de cinco pandas en el periodo 1965-1980.

Sin duda, el mejor ejemplo que ilustra el uso de la diplomacia panda e incluso se puede considerar el detonante de una nueva etapa es cuando en una cena de la histórica visita del presidente Richard Nixon a Pekín, en febrero de 1972, la primera dama Patricia Nixon mencionó su debilidad por los pandas gigantes al premier chino Zhou Enlai, quien deseoso por mejorar las relaciones con Estados Unidos ofreció obsequiarle unos. Una pareja de pandas llegaría a la capital estadounidense dos meses después, mismos que se convirtieron en un símbolo del acercamiento histórico entre la China de Mao y Estados Unidos, además de ser durante mucho tiempo uno de los mayores atractivos del Zoológico de Washington (los pandas murieron en 1992 y 1999). La diplomacia panda de 1972 sirvió como un importante factor que elevó el punto de vista del público americano de la República Popular China de una calificación favorable de $23 \%$ en 1972 a un $49 \%$ en 1973, mientras que disminuyó la opinión desfavorable en casi $30 \%$ en un año, allanando el camino para la normalización de las relaciones entre China y Estados Unidos en 1979 (Xing, 2010 p. 38).

De 1972 a 1982, otros 14 pandas fueron dados como regalos de buena voluntad a países capitalistas occidentales que establecieron relaciones diplomáticas con la República Popular China en este periodo: cuatro fueron entregados a Japón (1972, 1980 y 1982), un par a Francia (1973), a Gran Bretaña (1974) y Alemania (1974), a México (1975) y España (1978).

Como se puede observar, la mayoría de los países que recibieron pandas eran desarrollados en el momento y sólo dos en desarrollo. México (que se analizará con mayor detalle en el próximo apartado) y España, en este caso los pandas Shao Shao y Chang Chang fueron los primeros ejemplares que llegaron a España como regalo a los reyes durante su visita a China en 1978, 
y como el obsequio fue manejado a título personal, los monarcas los cedieron al Zoológico de Madrid.

El 8 de enero de 1981 China se adhirió a la Convención sobre el Comercio Internacional de Especies Amenazadas de Fauna y Flora Silvestres (CITES), quedando obligada a observarla a partir de abril de ese mismo año, por lo que quedó impedida para dar pandas a países extranjeros como regalo. Esto daría lugar al esquema bajo el cual China prestaría pandas como bienes comerciales a otros países.

En 1984 la Occidental Petroleum Corporation financió el traslado de un par de pandas al Zoológico de Los Ángeles en el marco de los Juegos Olímpicos a celebrarse en esa ciudad ese mismo año. Permanecieron en Los Ángeles durante 90 días y 30 más en el Zoológico de San Francisco. Este esquema meramente comercial incentivó a parques zoológicos de Norteamérica y Europa a obtener pandas en calidad de préstamo. ${ }^{3}$ Este enfoque fue blanco de duras críticas de organizaciones ambientalistas internacionales porque ponían en peligro la salud y la seguridad de los pandas. Esta crítica obligó a Beijing a dejar de prestar pandas sólo con fines comerciales.

Esta situación dio lugar al esquema actual enfocado en un marco del proyecto de investigación científica del panda. En 1993, la Asociación para la Conservación de la Vida Silvestre de China (CWCA) y la Asociación de Zoológicos de China alcanzaron un acuerdo con el organismo internacional de conservación de vida silvestre para dar en préstamo a parejas de pandas gigantes por un periodo de entre 10 y 15 años. Para proceder de tal forma, se debe suscribir un acuerdo entre la Asociación para la CWCA y los parques zoológicos extranjeros, que se comprometen a pagar un millón de dólares por año "para la conservación de los pandas gigantes en China". De esta cantidad, $70 \%$ se destina para ese señalado fin, 20\% lleva como destinatario el Centro de Investigación y Conservación para el Panda Gigante en Wolong, y el restante $10 \%$ es para "gastos del manejo del proyecto". El zoológico involucrado recibirá a cambio una pareja de pandas, estipulándose que habrá de pagarse una suma adicional de 600 mil dólares por cada cachorro de panda nacido en el zoológico, mismo que le pertenece a China y que debe ser devuelto en un

3. Nicholls sugiere que pareciera ser que los dos pandas eran parte del acuerdo que la gerencia de la Occidental Petroleum Corporation había firmado en 1984 con el gobierno de la República Popular China para desarrollar una mina de carbón a cielo abierto en la provincia de Shanxi y para lo cual la compañía con sede en Los Ángeles invertiría alrededor de 580 millones de dólares (Nicholls, 2010). 
periodo máximo de dos años. ${ }^{4} \mathrm{El}$ acuerdo establece también que el zoológico no puede disponer, sin previa autorización de la parte china, de muestras de material genético, ni transferirlas a terceras partes. Se estipula un seguro para la transportación de los pandas y en caso de fallecimiento de un panda de más de 18 meses o mayor atribuible a una negligencia del parque anfitrión, se deberá indemnizar a la parte china con 800,000 dólares. Se debe reportar a los chinos el monitoreo diario y mensual de la salud de los animales, los que serán sometidos a una revisión médica integral cada año. Finalmente, se establece que cualquier cuestión relacionada con el acuerdo se consultará de forma amistosa entre las partes y de no lograr acuerdo, se procedería a un juicio arbitral (The Smithsonian Institution, 2011).

En el caso de Tailandia, el Zoológico de Chiang Mai suscribió, en 2003, un contrato de alquiler de los pandas Chuang Chuang y Lin Hui que procrearon a Lin Ping, una hembra nacida en el zoológico tailandés y que se convirtió en una estrella de televisión. Como el panda debía volver a China en 2013, se inició un largo periodo de negociaciones, por las cuales se acordó que el panda Lin Hui podría permanecer en Tailandia durante 15 años más. El acuerdo fue suscrito en 2012 y, para concretarlo, el primer ministro tailandés Yingluck Shinawatra tuvo que intervenir, pidiendo al entonces premier chino Wen Jiabao considerara extender el contrato de préstamo del panda (Bangkok Post, 2012). La restricción de devolver los pandas nacidos en territorio fuera de China no existía en la etapa en que los pandas eran otorgados en donación, de hecho, nacieron y sobrevivieron ocho crías: cinco en México, dos en Tokio y una en Madrid (15 cachorros se malograron). Cabe resaltar que, jurídicamente, los dos pandas del Zoológico de Chapultepec son los únicos ejemplares no chinos del mundo.

Los montos a pagar han hecho que la diplomacia panda sea sobre todo aplicable a los países desarrollados: Estados Unidos, Corea del Sur, Tailandia, Austria, Australia, España y Japón son algunos de los países que han recurrido a este esquema para adquirir pandas.

Por otra parte, al ser un instrumento diplomático, no puede permanecer al margen de otras cuestiones diplomáticas: China concedió el préstamo por 10 años de los pandas Feng Yi y Fu Wa en 2012, en un intento de resaltar los 40 años de relaciones diplomáticas entre China y Malasia, con el fin de afianzar la floreciente relación económica entre los dos países, pero después

4. El acuerdo con el Zoológico de Washington establece cuatro años. 
de la desaparición del vuelo 370 de la Malaysia Airlines, la relación se tensó repentinamente. Unos 30,000 turistas chinos cancelaron sus planes de vacacionar en Malasia después del incidente, y el embajador chino Huang Huikang reprendió públicamente a las autoridades malasias por mostrarse demasiado "inexpertos y carentes de capacidad" para llevar a cabo la investigación correctamente (Taylor, 2014). Los pandas llegaron a Kuala Lumpur en mayo de 2014; en la ceremonia alusiva a la llegada de los pandas, participaron el ministro de Recursos Naturales y Medio Ambiente, G. Palanivel y el embajador chino en Malasia, este último declaró que "su llegada promoverá la cooperación en áreas tales como la silvicultura y la conservación de la biodiversidad entre nuestros dos países", agregando que también ayudaría a mejorar el mutuo entendimiento y la amistad entre los dos pueblos, escribiendo un nuevo capítulo en la historia de las relaciones amistosas entre China y Malasia (Jacobs, 2014). Los préstamos de osos pandas a Canadá, Francia y Australia coincidieron con acuerdos para la compra de uranio, que China necesita a fin de cumplir su meta de aumentar su capacidad nuclear para el año 2050. Beijing también le prestó dos pandas a Japón en 2011, y ambos países dijeron que esperaban que esto redujera la tensión generada por la disputa en torno a islas del Pacífico (Anderlini, 2017).

Aunque se ha determinado que no fue hasta el advenimiento del nuevo milenio que la diplomacia panda recobró su importancia para la política exterior de Beijing (Xing, 2010 p. 49), es evidente que con la llegada de Xi Jinping al poder, ésta ha retomado un impulso cada vez más creciente que no es fortuito: Xi sabe qué es el poder blando: en octubre de 2017, en su discurso de más de tres horas durante la apertura del xix Congreso del Partido Comunista en Beijing, con una alocución en la cual proclamó una nueva era en la política china, declaró:

[...] se ha promovido integralmente la diplomacia de gran país con peculiaridades chinas y se ha configurado una disposición diplomática omnidireccional, de múltiples niveles y multidimensional [...] Hemos propuesto estructurar una comunidad de destino de la humanidad y hemos impulsado el cambio del sistema de la gobernanza global. Nuestro país ha incrementado aún más su capacidad internacional de influir, inspirar y moldear, haciendo con ello nuevas e importantes contribuciones a la paz y el desarrollo mundiales [...] Promoveremos la construcción de nuestra capacidad de difusión internacional, contaremos bien lo que pasa en China y presentaremos una China real, multidimensional e integral, con vistas a acrecentar el poder cultural blando de nuestro país (Jinping, 2017). 
Tampoco desconoce que en este poder blando se circunscribe a la diplomacia pública y que la diplomacia panda es una expresión de esta última, por lo que está decidido a usar el hecho de que es exclusiva de China. Según Anderlini (2017), medios de comunicación estatales chinos describen al panda gigante como una de las más "poderosas armas" del presidente Xi en su esfuerzo por construir el poder blando. A través de Twitter, Facebook y YouTube (los cuales están bloqueados en China) los medios estatales bombardean con incontables videos de travesuras de pandas en un intento de hacerlos asociar con China a la audiencia global.

En marzo de 2013, Er Shun y Da Mao llegaron al Zoológico de Toronto en préstamo de China y permanecerían en el zoológico de Toronto hasta 2018, cuando se los trasladaría al zoológico de Calgary para establecerse ahí durante cinco años. Estos pandas procrearon a Jia Panpan (esperanza canadiense) y Jia Yueyue (alegría canadiense) que nacieron en el Zoológico de Toronto el 13 de octubre de 2015.

Los pandas Cai Tao (porcelana colorida) y Hu Chun (lago de primavera), de siete años de edad, llegaron a Yakarta el 28 de septiembre de 2017 a un zoológico safari en la isla de Java como parte de un acuerdo de cooperación entre la Reserva Natural Nacional de Wolong y el citado zoológico indonesio. Indonesia es el decimosexto país del mundo y el cuarto del sudeste asiático que recibe un préstamo de China para criar a pandas gigantes, según el safari situado en Bogor, a 50 kilómetros al sur de Yakarta (Lo Scalzo, 2017).

La diplomacia panda se convierte en una herramienta más de la actual administración china para posicionarse en el escenario global, "en la nueva era” que ha proclamado el presidente Xi Jinping. De la mano de Xi, el líder más poderoso del país en décadas, se quiere moldear el orden mundial para colocarse como referente, crear oportunidades estratégicas para sí y para sus empresas y legitimar su sistema de gobierno. Y ya no se recata en anunciarlo. "Nunca, desde los tiempos de Mao, un líder chino había contado con tanto poder, ni se había sentido tan seguro en el cargo" (Vidal, 2018). Además de la presencia con sus bancos enfocados en los temas del desarrollo, su Nueva Ruta de la Seda (se trata de la red de obras de infraestructura que implica varios corredores desde China hacia el oeste para conectar por tierra y por mar a China con países de Asia Central, Medio Oriente, África y Europa). "Su inversión en diplomacia ha sido vasta. En 2017 destinó a este fin 7,800 millones de dólares, un aumento del $60 \%$ respecto a 2013. Por contra, Estados Unidos ha propuesto recortar un $30 \%$ el gasto de su servicio exterior" (Vidal, 2018). 
Hasta mediados de 2015, había 42 pandas en parques zoológicos de 12 países fuera de China continental, según las estadísticas oficiales. Hoy en día hay 70 pandas en 20 países fuera de China y otros préstamos han alcanzado ya un acuerdo o están en proceso de negociación. En sólo los últimos meses China ha enviado - o prometió enviar - pandas a Alemania, Holanda, Finlandia, Dinamarca e Indonesia (Anderlini, 2017).

La diplomacia panda también sirve para lograr objetivos diplomáticos de largo y mediano alcance de China. Se trata de mantener una estrecha relación con su alianza y mantener en alto a China en las agendas diplomáticas y políticas de los países seleccionados, desactivar tensión con antiguos adversarios, así como allanar el camino para un cambio diplomático importante en la comunidad internacional y crear una relación más cooperativa, más estrecha para facilitar la reunificación nacional entre China continental y Taiwán ${ }^{5}$ (Xing, 2010 p. 26). "No se puede dar un panda a otra institución sin que los políticos sean involucrados”, dijo Nicholls (Davison, 2016). En el caso de la actual administración china, la razón más importante para el aumento de emisarios de panda es el énfasis del presidente Xi en mejorar el poder blando chino en el extranjero. Según varias personas con conocimiento del proceso, $\mathrm{Xi}$ se involucra personalmente en la negociación y firma de cada préstamo de panda a un país extranjero (Anderlini, 2017). Las decisiones acerca de alquilar pandas son tomadas al más alto nivel y con frecuencia se anuncian o implementan durante las visitas de Xi al extranjero (Szczudlik, 2017).

Implícito en la concesión de los pandas, es el entendimiento de que el país que los recibe reitera la aceptación de las posiciones políticas de China como que sólo el Partido Comunista tiene el derecho a gobernar el país (Anderlini, 2017), y que el Tíbet y Taiwán son parte integrante de China.

Según la especialista del tema Kathleen Buckingham, los pandas no son explícitamente ofrecidos a cambio de recursos. Es más sutil, dice: "El panda se utiliza para sellar simbólicamente el acuerdo y expresar la intención de una

5. En 2008 China dio a Taiwán dos pandas. Existieron intentos anteriores en 2001 y 2005 que fueron rechazados por los gobiernos taiwaneses proindependentistas. En 2008 los aceptaron, con la llegada al poder de un partido nacionalista que ha llamado para mejorar las relaciones con la China continental. A pesar de que los pandas fueron aceptados esta vez, no dejó de verse un signo de motivaciones políticas de China tras el regalo, pues los pandas fueron llamados "Tuan Tuan" y "Yuan Yuan", que combinados, "Tuan yuan", forman la frase que en chino significa reunión o unificación, provocando protestas en Taiwán (Szczudlik, 2017). En este caso, el gobierno chino decidió regalar a Taiwán dos pandas, sin contrato ni dinero de por medio. 
larga y próspera relación" (Hogenboom, 2013). Existen beneficios colaterales. Tras el préstamo de la pareja de pandas, Beijing y Edimburgo firmaron acuerdos por valor de US $\$ 4,000$ millones en las áreas de energía renovable, comercio de salmón y venta de vehículos Land Rover. Un portavoz del gobierno escocés le dijo a la BBC que "las exportaciones escocesas a China casi se han duplicado en los últimos cinco años, hasta alcanzar US $\$ 800$ millones. Fortalecer nuestras relaciones traerá beneficios sustanciales a ambos países. Queremos profundizar estos vínculos y establecer nuevas áreas de cooperación", señaló el portavoz. El estudio resalta que el acuerdo de Escocia representó una pérdida para Noruega, que por dos décadas había abastecido a China de la mayor parte de su consumo de salmón. Es probable, no obstante, que los pandas no tengan la culpa. Los investigadores señalan que quizás sea más bien porque Noruega le otorgó el Premio Nobel de la Paz al disidente chino Liu Xiaobo; desde entonces, las relaciones entre ambos países se han agriado (Hogenboom, 2013).

Difiriendo de este enfoque, Brînza (2016), vicepresidente del Instituto Rumano para el Estudio de Asia y el Pacífico afirma que actualmente los pandas no se utilizan tanto para atraer los ojos de los gobiernos. Aun así, el animal sigue siendo uno de los símbolos chinos más importantes y un gran ícono del poder blando chino. Sin embargo, en su parecer estas criaturas están siendo reemplazadas por otra herramienta diplomática, también con raíces históricas: la diplomacia de la Nueva Ruta de la Seda.

Por otra parte, cabe señalar que a finales de 2016 la Unión Internacional para la Conservación de la Naturaleza (UICN), ${ }^{6}$ la autoridad global sobre las especies en peligro de extinción, oficialmente cambió al panda de animal "en peligro" a "vulnerable", en lo que aparentemente fue una validación del esfuerzo de décadas de China para salvar a su símbolo nacional de la extinción, pero el gobierno chino no lo vio de esa manera. Supuestamente, funcionarios chinos se disgustaron con la decisión y han venido cabildeando para revertirlo. Las autoridades están preocupadas porque este cambio en el estatus del panda los convierte en objetos de menor valor, desde una perspectiva política

6. La Unión Internacional para la Conservación de la Naturaleza (UICN) es una asociación compuesta por Estados soberanos, agencias gubernamentales y organizaciones de la sociedad civil. Fue fundada en octubre de 1948 y su misión es "influenciar, alentar y ayudar a las sociedades de todo el mundo a conservar la integridad y diversidad de la naturaleza y asegurar que todo uso de los recursos naturales sea equitativo y ecológicamente sostenible". Tiene su sede en Gland, Suiza. 
y financiera, y que se podrían ver amenazados sus ingresos por turismo y en el negocio de préstamos de pandas a países extranjeros (Anderlini, 2017).

\section{El caso de México: relaciones diplomáticas y pandas}

México fue el único país de América Latina y, en su momento, uno de los dos países en desarrollo (si se considera así a España en 1978) que fueron destinatarios de la diplomacia panda de China.

No obstante no ser el primer país del continente en establecer relaciones con la República Popular China, pues ya lo habían hecho antes Cuba (1960), Canadá y Chile (1970), el contexto en que se dio y la situación geopolítica de esa época son los elementos que pudieron ser razones de la donación de un par de pandas al país, pero quizá más importante era el hecho de que "el país asiático necesitaba encontrar un camino hacia América Latina. La apertura de la Embajada mexicana era importante para la estrategia diplomática china, porque les permitía observar lo que ocurría en Norteamérica, Centroamérica e incluso Sudamérica" (López, 2016).

México y la República Popular China establecieron relaciones diplomáticas el 14 de febrero de 1972. El hecho tiene como antecedente el discurso que el 5 de octubre de 1971 el presidente mexicano Luis Echeverría Álvarez pronunció durante el debate general de la Asamblea General de Naciones Unidas, donde declaró que "la universalidad a la que aspira la ONU conocería un avance decisivo con el ingreso de la República Popular China. La soberanía y la integridad territorial de un Estado son jurídicamente indivisibles". Después que la Asamblea General aprobara la resolución para la "restitución de todos sus derechos y reconocer a los representantes de su gobierno como los únicos representantes legítimos de China en las Naciones Unidas", la Cancillería mexicana expidió un comunicado reconociendo al gobierno de Beijing como el único representante legal de China. En noviembre, el gobierno mexicano declaró por iniciativa propia "ruptura de relaciones diplomáticas" con Taiwán. 
El 10 de septiembre de 1975 arribaron al Zoológico de Chapultepec Pe Pe y Ying Ying, ${ }^{7}$ la pareja de pandas gigantes que el gobierno chino obsequió a México tras el establecimiento de relaciones diplomáticas. Los trajo el vicepremier del Consejo de Estado, Chen Yonggui y fueron recibidos por la primera dama, la "compañera" María Esther Zuno de Echeverría y Octavio Sentíes, regente del Distrito Federal. A la usanza de la época, se develó una placa alusiva con la leyenda "La República Popular de China, en noble gesto de amistad, donó al pueblo mexicano los dos ejemplares de oso panda que aquí se exhiben. Es un privilegio para el Zoológico de la Ciudad de México contar con tan bellos y excepcionales especímenes. 10 de septiembre de 1975".

México está en el umbral de quedarse sin pandas por la edad de los ejemplares que viven en Chapultepec, de hecho, los más longevos del mundo, pero la diplomacia panda no se agota: el 3 de junio de 2017, el embajador de China en México, Qiu Xiaoqi, participó en el evento "Homenaje a los Pandas de la Ciudad de México" a 42 años de la llegada de los pandas a México, llevado a cabo en el Zoológico de Chapultepec. El diplomático chino señaló que "los osos panda son los embajadores magníficos de amistad que unen a ambos países desde hace más de 40 años" y destacó que hoy en día las relaciones bilaterales están mejor que nunca, ya que México es un socio estratégico con excelentes relaciones políticas (Alcántara \& Jianrong, 2017).

Hasta la fecha se agradece en círculos oficiales y de prensa chinos al entonces presidente Luis Echeverría por el apoyo que dio para la recuperación del asiento de China (Anguiano, 2012: 39), éste fue un importante catalizador en el desarrollo de las relaciones sino-mexicanas, donde la diplomacia panda desempeñó un importante papel, así lo reconoce el embajador Qiu Xiaoqi (2017) en un artículo publicado en el diario El Sol de México, donde señalaría:

Los intercambios humanísticos y culturales han impulsado la apreciación mutua entre China y México. Éstos, que comenzaron con la "diplomacia del panda", se han fortalecido gracias a la admiración mutua y a la amistad entre sus pueblos. Debido al esfuerzo conjunto realizado por ambas partes en casi medio siglo.

7. En 1980, Pe Pe y Ying Ying tuvieron una primera cría, la primera nacida en cautiverio fuera de China, pero murió a los pocos días de nacida. El 21 de julio de 1981 nació Tohuí, la panda más famosa de México y tuvieron otros seis cachorros, de los cuales se lograron cuatro, entre ellos Shuan Shuan, que es el panda más longevo del mundo con 30 años (nació en 1987); Tohuí tuvo a Xin Xin. Shuan Shuan y Xin Xin son los únicos dos pandas que viven actualmente (marzo de 2018) en Chapultepec y son los únicos ejemplares del mundo que no pertenecen a China. 


\section{Conclusiones}

La diplomacia panda busca generar una opinión pública relativamente favorable o lo menos antagonista posible y a la vez obtener beneficios de los gobiernos de los países receptores de ejemplares de esta especie. Es una herramienta exclusiva de China que ha servido a su política exterior durante mucho tiempo, pero que ha cobrado importancia y vigor en la administración de Xi Jinping que reconoce y considera las ventajas que en el escenario internacional y, particularmente, en sus relaciones bilaterales puede proporcionarle. Sin duda

[...] la expansión de China en el planeta y el uso diplomático de los pandas es ahora más obvio y la motivación para hacer los préstamos cada vez es menos clara. No se trata sólo de su protección sino que están cada vez más vinculados con ambiciones políticas y económicas (Cheng, en Hogenboom, 2013).

México fue objeto de la práctica de la diplomacia panda en la década de los setenta del siglo pasado. China reconocía con ello el papel de este país en el proceso de restitución de sus derechos en Naciones Unidas y quizá lo consideraba como la puerta para obtener el reconocimiento de Latinoamérica, lo cual, demuestran los hechos, no sucedió en la medida de las aspiraciones chinas, que aún hoy en día carecen del reconocimiento de algunos de los países centroamericanos y de Paraguay. miv

\section{Referencias bibliográficas}

Alcántara, E. \& Jianrong, P. (2017). ESPECIAL: China reconoce a México por cuidado y conservación de osos panda. Xinhua Español. Recuperado de http://spanish.xinhuanet.com/2017-06/04/c_136337916.htm Fecha de consulta: 8 de marzo de 2018.

Anderlini, J. (2 de noviembre de 2017). How the panda became China's diplomatic weapon of choice. Financial Times. Recuperado de https:// www.ft.com/content/8a04a532-be92-11e7-9836-b25f8adaa111 Fecha de consulta: 17 de febrero de 2018 .

Anguiano, E. (2012). Sin sustento político, imposible construir relaciones económicas bilaterales sólidas. En Dusell, E. (Coordinador). Cuarenta años de la relación entre México y China: acuerdos, desencuentros y futuro, 37-47. 
Asamblea General (1971). Resolución 26/2758 Restitución de los legítimos derechos de la República Popular China en las Naciones Unidas. Nueva York: Naciones Unidas.

Bangkok Post. (21 de noviembre de 2012). "Thailand, China sign four MoUs". Recuperado de https://www.bangkokpost.com/news/topstories/322412/ favicon.ico Fecha de consulta: 30 de enero de 2019.

Brînza, A. (30 de septiembre de 2016). How China has gone from panda diplomacy to New Silk Road smart power. South China Morning Post. Recuperado de http://www.scmp.com/comment/insight-opinion/article/2023656/ how-china-has-gone-panda-diplomacy-new-silk-road-smart-power Fecha de consulta: 13 de febrero de 2017.

Buckingham, K., Neil, J., David, W., \& Jepson, P. (2013). Diplomats and Refugees: Panda Diplomacy, Soft "Cuddly" Power, and the New Trajectory in Panda Conservation. Environmental Practice. 1-9. doi:10.10170S1466046613000185

Cámara de Diputados. (2006). Informes Presidenciales. Luis Echeverría Álvarez. (RE-ISS-09-06-14). México, D.F. México: Dirección de Servicios de Investigación y Análisis. Recuperado de http://www.diputados.gob.mx/sedia/ sia/re/RE-ISS-09-06-14.pdf

Cull, N. (2009). Diplomacia Pública: consideraciones teóricas. Revista Mexicana de Política Exterior, 85, 55-92. Recuperado de https://revistadigital.sre. gob.mx/images/stories/numeros/n85/cull.pdf

Davison, J. (12 de marzo de 2016). 'Cuddly' diplomacy: Canada's new panda cubs renew the politics of cuteness. CBCNews. Recuperado de http://www. cbc.ca/news/canada/giant-panda-cubs-politics-economics-conservationtoronto-zoo-1.3481839 Fecha de consulta: 13 de febrero de 2017.

Espinosa de los Monteros, C. (2012) Prólogo. La Marca España: una empresa de todos. Retos de nuestra acción exterior: Diplomacia Pública y Marca España. (pp. 15-16). Madrid, España: Escuela Diplomática.

García de Alba, C. (2009). Diplomacia Pública, propaganda y poder blando. Revista Mexicana de Política Exterior, 85, 221-228.

Hogenboom, M. (5 de octubre de 2013). Una nueva fase de la "diplomacia panda" china. $B B C$. Recuperado de https://www.bbc.com/mundo/noticias/2013/10/130927_china_diplomacia_panda_finde_mes

Itamaraty. (30 de mayo de 2015). Ministério das Relações Exteriores. Diplomacia Pública. Recuperado de http://www.itamaraty.gov.br/pt-BR/ diplomacia-publica Fecha de consulta: 23 de febrero de 2017. 
Jacobs, J. (21 de mayo de 2014). Feng Yi \& Fu Wa Arrived in Malaysia. Giant Panda Global. Recuperado de http://www.giantpandaglobal.com/zoo/ zoo-negara-malaysia/feng-yi-fu-wa-arrived-in-malaysia/ Fecha de consulta: 5 de marzo de 2018.

Jinping, X. (18 de octubre de 2017). Texto íntegro del informe presentado por Xi Jinping ante XIX Congreso Nacional del PCCh. Xinhua. Recuperado de http://spanish.xinhuanet.com/2017-11/03/c_136726335.htm Fecha de consulta: 20 de febrero de 2018.

Lin,W. (6 de febrero de 2009). China's Panda Diplomacy. Mainland Affairs Council. Recuperado de http://www.mac.gov.tw/public/Attachment/052716233690.pdf Fecha de consulta: 13 de febrero de 2017.

López, R. (4 de julio de 2016). Entrevista con Eugenio Anguiano, el primer embajador de México en China. UNAM-China. Centro de estudios mexicanos. Recuperado de https://unamenchina.net/2016/07/04/entrevistacon-eugenio-anguiano-el-primer-embajador-de-mexico-en-china/ Fecha de consulta: 23 de febrero de 2018.

Lo Scalzo, J. (28 de septiembre de 2017). Dos osos panda viajan, como embajadores, de China a Indonesia. Agencia EFE. Recuperado de http:// www.efeverde.com/noticias/zoologico-indonesio-recibe-a-dos-pandasgigantes-chinos/ Fecha de consulta: 10 de febrero de 2018.

Metzl. J.F. (1999). Popular Diplomacy. Daedalus, 128(2): 177-192. Cambridge, United States: The MIT Press.

Nicholls, H. (2010) The Way of the Panda: The Curious History of China's Political Animal. London, United Kingdom: Profile Books.

Nye, J. (2010). "Prefacio y Capítulo 5 'El poder blando y la política exterior americana', en Soft Power, Public Affairs, New Hampshire, 2004, pp. IXXIII y 127-147". Relaciones Internacionales, 14, 117-140. Recuperado de https://repositorio.uam.es/bitstream/handle/10486/678144/RI_14_7. pdf? sequence $=1$

Russo, N. (6 de junio de 2016). "Koalas are Australia's Most Effective Diplomats. Atlas Obscura. Recuperado de https://www.atlasobscura.com/articles/koalas-are-australias-most-effective-diplomats. Fecha de consulta: 28 de febrero de 2018

Taylor, A. (21 de mayo de 2014) China, Malaysia and the weird world of panda diplomacy. Washington Post. Recuperado de https://www.washingtonpost. com/news/worldviews/wp/2014/05/21/china-malaysia-and-the-weird- 
world-of-panda-diplomacy/?utm_term=.d400bb812f7d Fecha de consulta: 13 de febrero de 2017

The Smithsonian Institution (2011). Agreement between the Smithsonian Institution (SI) National Zoological Park and the China Wildlife Conservation Association (CWCA) concerning the giant pandas. Recuperado de http://www.governmentattic.org/11docs/SI-NZP-CWCAagreePandas_2011.pdf Fecha de consulta: 6 de marzo de 2018

Szczudlik, J. (11 de septiembre de 2017). "Role of 'Panda Diplomacy' in China's Foreign Policy”, Bulletin, 83(1023). Varsovia, Polonia: The Polish Institute of International Affairs. Recuperado de https://www.pism.pl/ publications/bulletin/no-83-1023. Fecha de consulta: 6 de marzo de 2018. Vidal, M. (3 de marzo de 2018). Así quiere China dominar el mundo. El País. Recuperado de https://elpais.com/internacional/2018/03/02/actualidad/1519993755_786257.html Fecha de consulta: 4 de marzo de 2018. Xiaoqi, Q. (11 de febrero de 2017). "Tender un puente de la amistad ChinaMéxico hacia el futuro”. Embajada de la República Popular China en México. Recuperado de http://mx.china-embassy.org/esp/ojos/t1437606. htm Fecha de consulta: 29 de marzo de 2018. 